\title{
EXPERIENCE OF CONTINUOUS AMBULATORY PERITONEAL DIALYSIS IN CHILDREN WITH CHRONIC KIDNEY DISEASE STAGE" V
}

\author{
AFROZ $\mathrm{S}^{1}$, FERDAUS $\mathrm{T}^{2}$, KHANAM $^{3}$, SIMI MA ${ }^{4}$, SHARMIM $\mathrm{S}^{5}$, HOSSAIN $^{6}$, RAHMAN MM ${ }^{7}$
}

\begin{abstract}
:
The lifespan and outcome of End Stage Kidney Failure (ESKF) children have dramatically improved since the development of continuous ambulatory peritoneal dialysis (CAPD), it offers several advantages over hemodialysis. This study was done to evaluate the efficacy, outcome and to find out the complication profile of CAPD. This prospective longitudinal study was carried out in the department of Pediatric Nephrology, Dhaka Medical College Hospital (DMCH), Bangladesh, over a period of 3 year. A total of 8 children with ESKF were included. (Age 5-14 year, $M: F=1: 1)$, All underwent CAPD. Average duration of CAPD 40.6 months with a total of 185 patient months of CAPD. The rate of complications was recorded. Common complications being observed were peritonitis 1 episode per 13.9 patient months, catheter obstruction by omental capture was in 4. Catheter tip dislocation was found in 3 children and all needed laparotomy and omentectomy. Satisfactory level of improvement of mean weight, mean serum albumin and declining of serum creatinine has been found after CAPD in all 8 study children. At the end of the study, 3 out of 8 are still on CAPD, 2 transferred to haemodialysis and 3 expired due to uncontrolled hypertension with congestive heart failure and sepsis. So, CAPD is an effective modality of renal replacement therapy for children. Early detection of complications and prompt therapy is essential for a favourable outcome. Placement of catheter by surgical method with elective omentectomy will reduce catheter related complications.
\end{abstract}

Key Words: Continuous ambulatory peritoneal dialysis (CAPD), Peritonitis, Children.

J Dhaka Med Coll. 2016; 25(1) : 32-38

\section{Introduction:}

Children constitute about $1-2 \%$ of total End Stage Kidney Failure (ESKF) population. The lifespan and outcome of ESKF children have dramatically improved since the development of renal replacement therapy (RRT) ${ }^{1}$. Continuous ambulatory peritoneal dialysis (CAPD) has been used in children since 1978. CAPD is an effective bridge to renal transplant in children with end stage renal disease ${ }^{2}$. It offers several advantages over haemodialysis (HD). CAPD is preferred over HD in children to avoid the problems of vascular access, anticoagulation and haemodynamic imbalance $^{3}$. In addition, CAPD is associated with better control of hypertension and correction of acid-base and fluid imbalances ${ }^{4}$. Despite advances in peritoneal dialysis (PD) systems, peritonitis is a significant clinical problem $^{5}$. There are two methods of CAPD catheter insertion. Surgical method of catheter insertion is performed by surgeons under general anaesthesia (G/A). Percutaneous technique is performed by nephrologists, which is minimally invasive, reliable, safe and costeffective method ${ }^{6}$. Very few published data regarding CAPD experience in children has been found ${ }^{2,3,6}$. So this study was done to evaluate the efficacy of CAPD in children, to find out the complication profile of CAPD in children.

\section{Methodology:}

This was a longitudinal interventional study with prospective follow up of the study subjects. It was conducted in the Pediatric Nephrology ward of Dhaka Medical College \& Hospital (DMCH), Dhaka, Bangladesh, over a period of 3 years. Children with CKD"V were selected

1. Dr. Shireen Afroz, Associate Professor of Paediatric Nephrology, BICH \& Dhaka Shishu (Children) Hospital, Sher e bangle Nagar, Dhaka- 1207

2. Dr. Tahmina Ferdaus, Medical Officer, Dhaka Shishu (Children) Hospital

3. Dr. Rokeya Khanam, Assistant Professor, Department of Paediatrics, DMCH

4. Dr. Maliha Alam Simi, Registrar, Department of Paediatrics, DMCH

5. Dr. Sanjida Sharmim, Medical Officer, Department of Paediatrics, DMCH

6. Dr. Nasir Hossain, Assistant Professor, Department of Paediatric Nephrology, DMCH

7. Dr. M Mizanur Rahman, Associate Professor, Department of Urology, DMCH

Correspondence : Dr. Shireen Afroz1, Associate Professor of Paediatric Nephrology, BICH \& Dhaka Shishu (Children) Hospital, Sher e bangle Nagar, Dhaka- 1207, safroz16@live.com, Cell 01715054239

Received: 15 March 2016

Accepted: 20 March 2016 
purposively. A total of 8 children with ESKF were included. (Age 5-14 year, $\mathrm{M}: \mathrm{F}=1: 1$ ) All underwent CAPD, The rate of complications and their outcome were recorded. The diagnosis of peritonitis was based on any 2 of the following criteria, symptoms of peritoneal inflammation- abdominal pain; cloudy fluid, with a total leukocyte count of more than 100 cell and $>50 \%$ neutrophils or presence of organism on gram staining or culture of CAPD fluid $^{7}$. Tunel infection was ensured clinically by the presence of sign's of inflammation around subcutaneous tunel and or purulent discharge from exit site ${ }^{2,7}$. Catheter insertion was done by 2 methods. Surgical catheter implantation was done by an urologist.

It was done under general anaesthesia with a small paraumbillical incision catheter was introduced inside abdomen and elective omentectomy was done in 2 children under 8 year of age. Finally through a subcutaneous tunnel, catheter was negotiated outside at the exit site. Percutaneous catheter was implanted by the pediatric nephrologist (author herself). With all aseptic precaution and under deep sedation and local anaesthesia at first abdomen
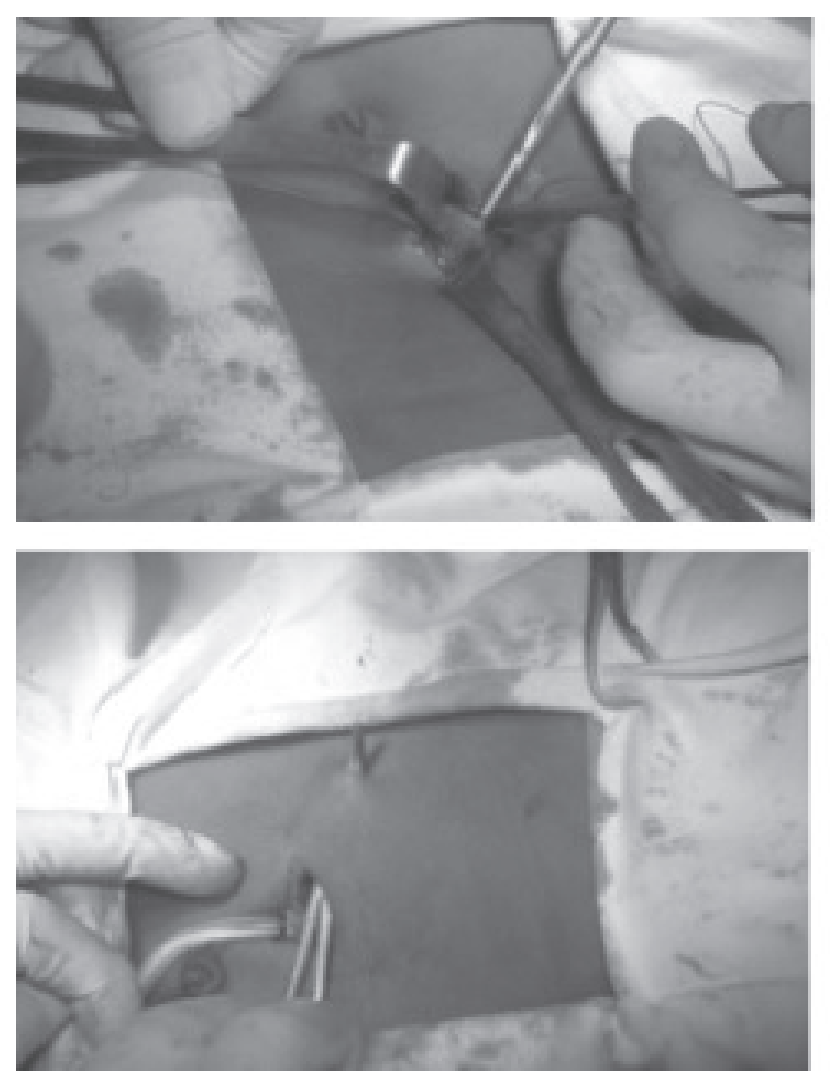

Procedure of surgically placed CAPD catheter was filled with normal saline through a wide bore needle, then a guide wire was introduced through the needle. Under the guidance of guide wire a wide bore dilator was introduced and the catheter was negotiated through the easily peel off tube inside abdomen. Free flow of fluid has been checked. Then through a subcutaneous tunnel the proximal end of the catheter was fixed at the exit site. To ensure the efficacy of CAPD we have compared the pre and post CAPD mean weight, mean serum albumin and mean serum creatinine of the study children. During follow up, catheter related complications like catheter blockage or catheter tip dislocation were confirmed by ultrasonography, plain x-ray abdomen or under $\mathrm{C}$ - arm.

Prior permission was taken and written informed consent was obtained from each parents. All precautions were taken to protect the anonymity of the participating subjects. As it was a hospital based study, so ethical issues related to surgical interventions were maintained as per hospital standard rules. Data were processed by using software SPSS 16. The test statistics used to analyze the data were chi square and student t test, $\mathrm{p}$ value < 0.05 was considered significant.
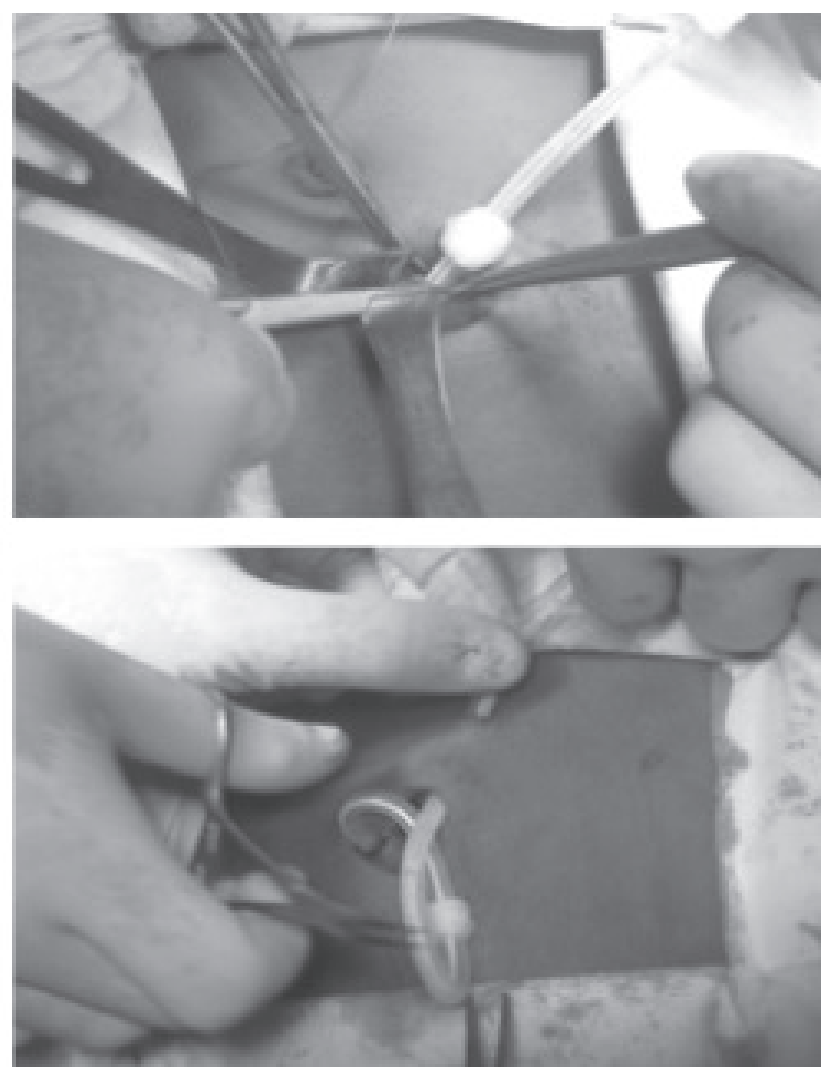

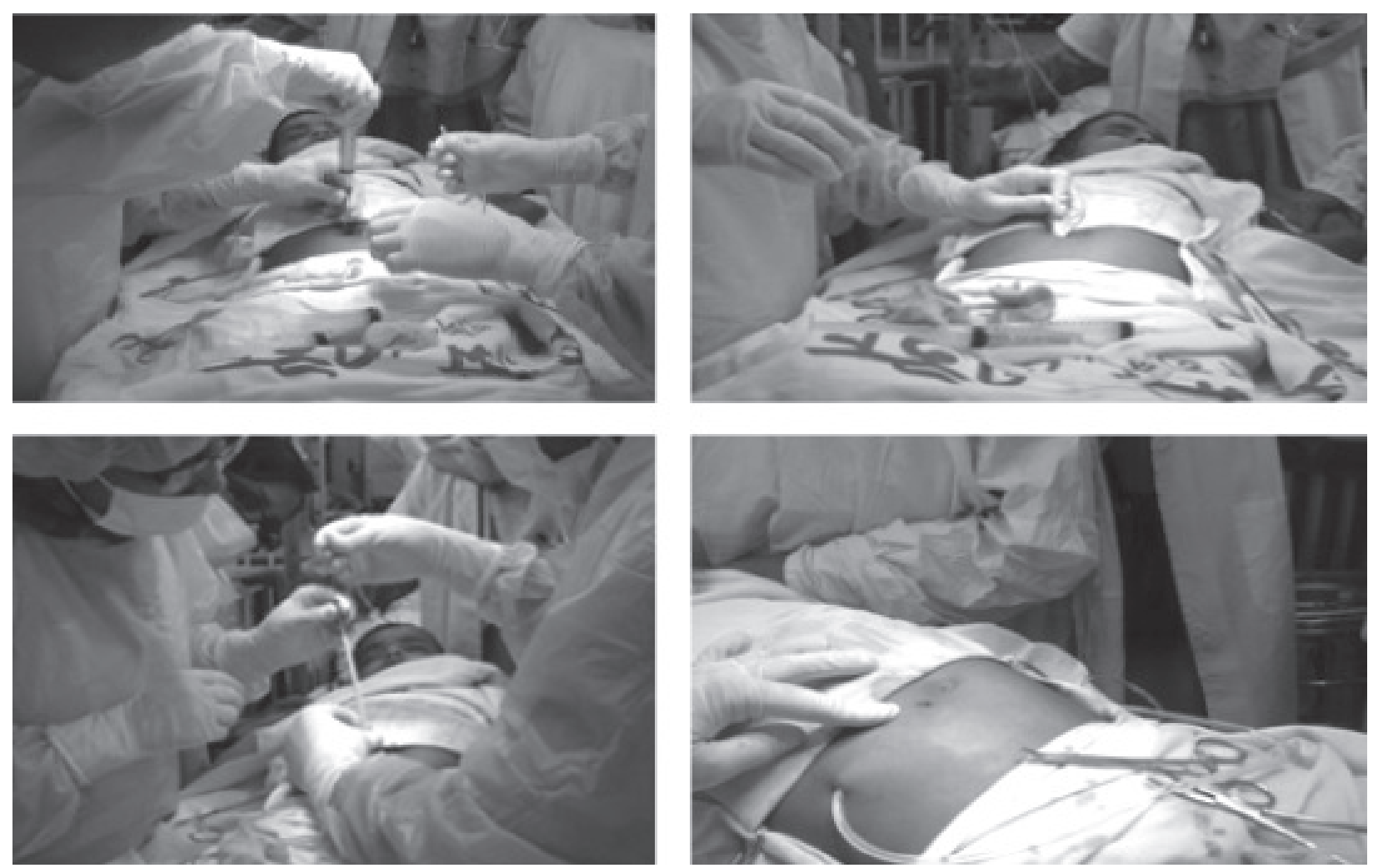

Procedure of percutaneously placed CAPD catheter

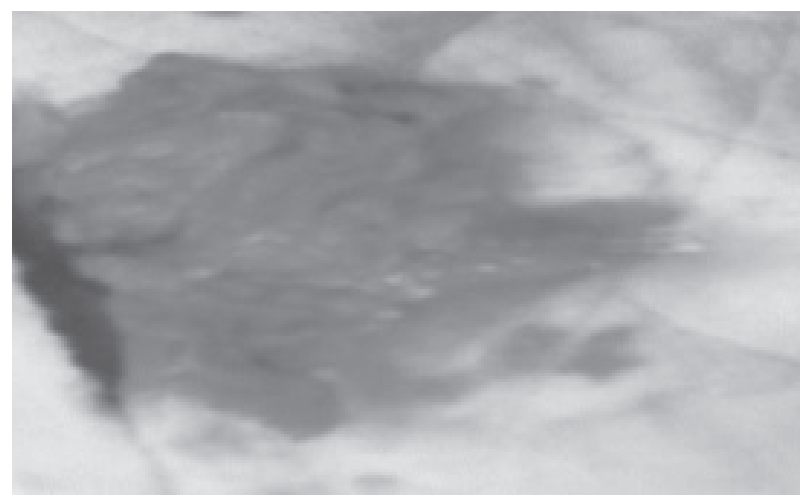

Complications: Omental wrapping needed laparptomy and omentectomy

Out of 8 cases posterior urethral valves, reflux nephropathy due to recurrent UTI, single hypoplastic kidney, poly cystic kidney disease were the underlying cause of CKD, no cause was identified in 3 cases they were admitted at late stage with small kidney and had no history suggestive of glomerulonephritis or any. Most probably congenital hypoplasia or dysplasia might be the cause. Another 1 with bilateral primary VUR. (table-I).

Table II

Socio demographic data of CKD cases $(n=8)$

\section{Results}

Table-I

Cause of CKD of the study cases.

\begin{tabular}{lc}
\hline Causes of CKD & Number \\
\hline Posterior urethral valve & 01 \\
Poly cystic kidney disease & 01 \\
Recurrent UTI with reflux nephropathy & 01 \\
Single hypoplastic kidney & 01 \\
Unknown & 03 \\
Bilateral primary VUR & 01 \\
\hline Total & 08 \\
\hline
\end{tabular}

\begin{tabular}{lcc}
\hline Age (year) & $5-8$ & Years 3 \\
\hline \multirow{3}{*}{ Sex } & $>8$ years & 5 \\
& M & 4 \\
Residence & F & 4 \\
& Urban & 5 \\
Socio economic & Rural & 3 \\
status & Middle class & 3 \\
& Lower middle class & 5 \\
\hline
\end{tabular}

Sociodemographic data showed that (table-II), all study patients were older than 5 year and majority were above 8year of age. Most of them 
were from urban areas. Majority were from middle and lower middle class family.

Table III

Procedure \& prescription

\begin{tabular}{lc}
\hline Procedure \& prescription & No \\
\hline Four exchanges per day & 08 \\
Single bag for single exchange & 02 \\
Single bag for 4 exchange & 06 \\
Average duration of & 40.6 \\
CAPD months & (Range 1-54 \\
& months) \\
Elective omentectomy during & 02 \\
catheter lacement & \\
Catheter replacement after 2 year & 02 \\
\hline
\end{tabular}

All patients were prescribed 4 sessions of CAPD per day with 4 hours dwell time. Among them single bag users were only 2 . Single bag for multiple exchanges were 6. Average CAPD duration in months 40.6. Elective omentectomy was done in 2 children under 8 year age. In 2 patients catheter replacement was needed after 2 year (table III).

\section{Table IV}

Complications of CAPD

\begin{tabular}{|c|c|}
\hline Complications & No. \\
\hline Peritonitis 1 episode/ pt month & $\begin{array}{l}1 \text { episode } \\
\text { per } 19.17 \\
\text { pt months }\end{array}$ \\
\hline $\begin{array}{l}\text { Catheter obstruction by } \\
\text { omental capture }\end{array}$ & 04 \\
\hline Catheter tip dislocation & 03 \\
\hline Tunel infection & 01 \\
\hline Inguinal hernia & 01 \\
\hline
\end{tabular}

Higher frequency of peritonitis being observed in this study. Catheter obstruction by omental capture and catheter tip dislocation were found in 4 and 3 cases. Tunel infection and inguinal hernia were also found (table-IV).

Table V

Complications of CAPD in surgical Vs percutaneously placed catheter

\begin{tabular}{|c|c|c|c|}
\hline Complications & $\begin{array}{l}\text { Surgically placed } \\
\text { catheter }(\mathrm{n}=5)\end{array}$ & $\begin{array}{l}\text { Percutaneously } \\
\text { placed catheter }(n=3)\end{array}$ & $\mathrm{P}$ value \\
\hline $\begin{array}{l}\text { Peritonitis } 1 \\
\text { episode/ pt month }\end{array}$ & $\begin{array}{l}1 \text { episode per } 17.6 \\
\text { pt months }\end{array}$ & $\begin{array}{l}1 \text { episode per } 1.57 \mathrm{pt} \\
\text { months }\end{array}$ & $<0.001$ \\
\hline Catheter obstruction by omental capture & 01 & 03 & 0.14 \\
\hline Catheter tip dislocation & 0 & 3 & 0.01 \\
\hline Tunelitis & 01 & 0 & ns \\
\hline Inguinal hernia & 01 & 0 & $\mathrm{~ns}$ \\
\hline
\end{tabular}

Significantly higher number of peritonitis being observed in children with surgically placed catheter $(\mathrm{p}<0.001)$. Catheter obstruction by omental capture and catheter tip dislocation were found in $100 \%$ cases of percutaneously placed catheter $(\mathrm{p}<0.01)$. Tunelitis and inguinal hernia were also found.

Table VI

Outcome of children on CAPD

\begin{tabular}{lc}
\hline Outcome & No. \\
\hline On CAPD & 03 \\
Transfer to HD & 02 \\
Kidney transplantation & 0 \\
Expired & 03 \\
\hline
\end{tabular}

At the end of this study it was found that 3 patients were still on CAPD, 2 transferred to haemodialysis for non effective clearance, and 3 expired as a complications of hypertensive encephalopathy with congestive heart failure and sepsis.

To ensure the efficacy of CAPD we have compared the pre and post CAPD mean weight, mean serum albumin and mean serum 
creatinine of the study children, which showed satisfactory level of improvement of weight, nutritional status (serum albumin) and declining of serum creatinine. (figure-1).

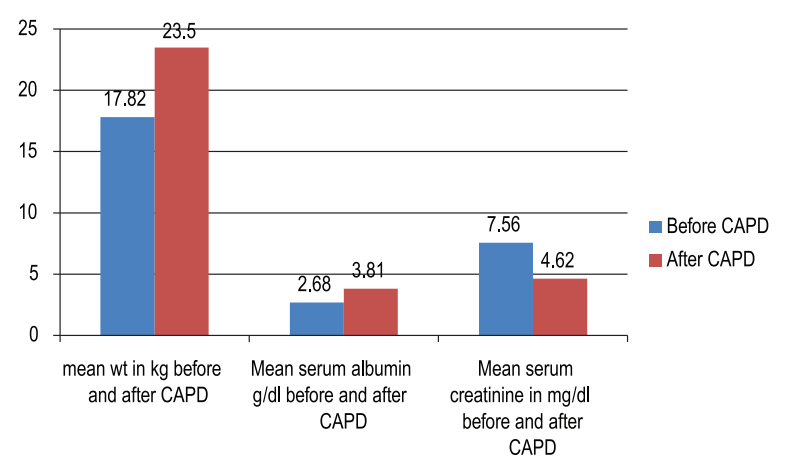

Fig.-1 Efficacy of CAPD

\section{Discussion:}

CAPD is the most common dialysis treatment modality used to treat pediatric patients with end-stage renal disease (ESRD), particularly in children less than five years of age $e^{1,2,6,8}$. CAPD offers several advantages over hemodialysis (HD) that are beneficial for children; however, there are clinical settings in which CAPD is contraindicated or may not be the best dialytic modality. In addition, although the principles of CAPD are the same for pediatric and adult patients, there are aspects of CAPD that are unique to children and infants, which need to be addressed to ensure the adequacy of dialysis and to reduce complications for the pediatric patient. In this prospective study, we evaluated the causative factors, outcomes, efficacy and complications of therapy in 8 patients. Posterior urethral valves, reflux nephropathy due to recurrent UTI, single hypoplastic kidney, poly cystic kidney disease were the underlying cause of CKD. In 2 cases no cause was identified, they were admitted at late stage with small kidney and had no history suggestive of glomerulonephritis or any. Most probably congenital hypoplasia or dysplasia might be the cause. Another 1 with bilateral primary VUR. Sociodemographic data showed that all study patients were older than 5 year and majority were above 8year of age. Most of them were from urban areas and from middle and lower middle class family. $\mathrm{DMCH}$ is a government public hospital so most of our study patients were from middle or lower middle class family and due to ignorance and financial constraint the parents brought their child at late stages. As CAPD cost is high they used single bag for 4 exchanges which might be an important cause of increased frequency of peritonitis. Moreover maintenance of sterility was an important factor for infection, which was not maintained strictly by the poor parent. All patients were prescribed 4 sessions of CAPD per day with 4 hours dwell time. Among them single bag users were only 2. Single bag for multiple exchanges were 6. Average CAPD duration in months 40.6 . Elective omentectomy was done in 2 children under 8 year age of group-II. In 2 patients catheter replacement was needed after 2 year.

At the end of this study it was found that 3 patient were still on CAPD, 2 transferred to haemodialysis for non effective clearance, and 3 expired as a complications of hypertensive encephalopathy with congestive heart failure and sepsis. Annabelle Chua et al. showed, 9 patients switched to ambulatory peritoneal dialysis (APD) during the follow-up period. The most common cause leading to end-stage renal disease (ESRD) in the patients was reflux nephropathy (22.9\%). The major complication during therapy was peritonitis, with 41 episodes seen in 17 patients ( 1 episode per 18 patientmonths). Of the children on APD, 7 developed 17 episodes of peritonitis (1 episode per 8.3 patient-months); of the children on CAPD, 10 developed 24 peritonitis attacks ( 1 episode per 24.9 patient-months). The other complications were inguinal hernia in 3 patients, subcutaneous leak in 4 patients, dialysate leak in 2 patients, pericardial effusion in 1 patient, umbilical hernia in 1 patient, hydrothorax in 1 patient, and cuff protrusion in 3 patients. During the follow-up period, 4 patients died owing to sepsis or cardiopulmonary complications. Only 1 patient was transferred to hemodialysis (owing to persistent Candida peritonitis). Experience of the present study also correlates with Annabelle Chua et al. ${ }^{9}$ that CAPD therapy is a good choice of treatment modality in the management of children with ESRD.

In this survey, significantly higher number of peritonitis being observed in percutaneously 
placed catheter $(\mathrm{p}<0.001)$. Omental wrap, poor catheter survival and more complications in young children less than 8 year has been found. But better catheter survival was observed by Rinadi et al. ${ }^{8}$ especially in younger children $(<$ 2 years), a group that previously had a decreased catheter survival rate compared to older age groups. They added that, in addition to the progressive increase in experience acquired by dialysis centers, this upward trend may also be related to greater use of doublecuffed catheters, with paramedian exit site, and a higher frequency of omentectomy ${ }^{8}$. So surgically placed catheter with elective omentectomy might be the cause of improved catheter survival. Infections of the peritoneal space are the main complication of $\mathrm{PD}$; they are usually caused by staphylococcal species. By comparison, fungi are responsible for 2 to 13 percent of such cases. Most fungal PDassociated infections are due to Candida species, especially C. albicans and C parapsilosis. Assorted molds and yeasts, such as species of Aspergillus ${ }^{9}$.

In the present study there was satisfactory level of improvement of weight, nutritional status (serum albumin) and declining of serum creatinine after CAPD (figure-1). There have not been any comparative studies of peritoneal dialysis (PD) and hemodialysis (HD) outcomes in children with end-stage renal disease (ESRD) to suggest superiority of one procedure versus the other. While the majority of pediatric patients with ESRD who require dialysis can be managed with chronic peritoneal dialysis (CPD), the choice of dialysis modality is most often based on patient and family preference, center philosophy, and availability of the desired modality ${ }^{8}$.

The quality of life for both patient and family assumes great importance in the selection of home dialysis therapy as a result of the "burden of care" associated with this approach to ESRD management. As such, careful evaluation of the family's social, psychological, and economic background, ideally by a multi professional team including the family physician and nephrologist, dialysis nurse, psychologist, and social worker, is mandatory if a fully informed decision regarding modality selection is to be made $^{10,12}$.

Survival rate of children younger than 20 years on chronic RRT has increased over the past years and varies from $79 \%$ to $82 \%$ at 10 years in developed countries. In this study that included children younger than 16 years, only $67 \%$ of patients were alive at the end of study period. It should be noted that a younger age at onset of RRT is a considerable mortality risk factor in all studies. Moreover, higher mortality in our study may be attributed to prolonged stay on dialysis and some socioeconomic factors. In some countries such as Jamaica and Nigeria, mortality rate of ESRD children is even higher than our results and is about $44.4 \%$ and $47 \%$, respectively ${ }^{10}, 11,12$.

\section{Conclusion:}

CAPD is an effective modality of RRT for children. Early detection of complications especially peritonitis and prompt therapy is essential for a favourable outcome. So surgically placed catheter with elective omentectomy might be the cause of improved catheter survival.

\section{Limitations:}

High cost of transplantation results in a long staying time on dialysis that impairs the quality of life in our patients. Limited welleducated nursing and social workers and lack of technical support restrict employment of CAPD in our area. Other studies are necessary to evaluate the long-term outcome of CAPD treatment in children with CKD-V.

\section{Acknowledgement:}

Our special thanks to Associate Professor Dr. M Mizanur Rahman, Department of Urology, Dhaka Medical College \& Hospital for whole hearted support for surgical catheter placement and management of catheter related complications.

\section{References:}

1. Waradi BA, Chadha V. Chronic Kidney disease in children: the global perspective. Pediatr Nephrol. 2007; 22(12): 1999-2009.

2. Kanitkar M, Ramamurthy HR. An Initial Experience of Continuous Peritoneal Dialysis in Children in the Armed Forces. MJAFI. 2007; 63: 220-222 
3. Alexander SR, Salusky IB, Warady BA, Watkins SL. Peritoneal dialysis workshop: Pediatric Recommendations. Perit Dial Int. 1997;17(Suppl 3): $25 \mathrm{~S}-27 \mathrm{~S}$.

4. Lingens N, Soergel M, Loirat C, Lemmer B, Scharer K. Ambulatory blood pressure monitoring in pediatric patients treated by regular haemodialysis and peritoneal dialysis. Pediatr Nephrol 1995; 9: 167-72.

5. Furth SL, Donaldson LA, Sullivan EK, Watkins SL. Peritoneal dialysis catheter infections and peritonitis in children: a reportof the North American Pediatric Renal Transplant Cooperative Study. Pediatr Nephrol 2000;15: 179-82.

6. Aksu N, Yavascan O, Anil M, Kara OD, Efdogan $\mathrm{H}$, Bal Al. A ten-year single centre experience in children on chronic peritoneal dialysissignificance of percutaneous placement of peritonel dialysis catheter. Nephrol Dial Transplant.2007 Jul;22(7):2045-51. Epub 2007.

7. Oreuopolus DG, Vas SI. Peritonitis in continuous ambulatory peritoneal dialysis: making therapeutic decisions easier. Arch Int Med 1987;147; 818-9.

8. Rinaldi S,et al. Chronic peritoneal dialysis catheters in children: a fifteen-year experience of the Italian Registry of Pediatric Chronic Peritoneal Dialysis. Perit Dial Int 2004; (24): 481-486

9. Annabelle Chua, MDBradly A, Warrady MD, Patric NiaudetMD, Melani Skim MD. Chronic peritoneal dialysis in children. Adv Perit Dial. 2003;19: 269-72.

10. Dönmez O, Aladag S, Cigerdelen N, Kocak S. Outcome in children on chronic peritoneal dialysis. Indian J Nephrol. 2012 ; 22(2): 94-97.

11. Mortazavi F, Maleki M. Management and outcome of children with end-stage renal disease in northwest Iran, Cochrane Database Syst Rev. 2007 Apr 18;(2):CD006515.

12. Prasad N, Gulati S, Gupta A, Sharma RK, Kumar A, Kumar R, et al. Continuous peritoneal dialysis in children: a single-centre experience in a developing country. Pediatr Nephrol 2006 ;21: 403-7. 